\title{
The Riemann Spring
}

\author{
Bohua Sun ${ }^{1}$ \\ ${ }^{1}$ Cape Peninsula University of Technology, Cape Town, South Africa*
}

(Dated: October 1, 2018)

\begin{abstract}
This paper attempts to propose a Riemann spring model via an analogy between the Riemann Zeta function of a complex number and the elastic spring in series. It is assumed that the Riemann spring is consist of infinite springs in series, whose $n$-th spring has elastic complex stiffness $k_{n}=n^{s}$, therefore total equivalent stiffness $K(s)$ is given by
\end{abstract}

$$
\frac{1}{K(s)}=\sum_{n=1}^{\infty} \frac{1}{k_{n}}=\sum_{n=1}^{\infty} \frac{1}{n^{s}}=\zeta(s)
$$

where the Riemann Zeta function is $\zeta(s)=\sum_{n=1}^{\infty} \frac{1}{n^{s}}$. The Riemann Hypothesis generalized variational is formulated by Lagrange multipliers.

Keywords: Riemann Zeta function, matrix, tensor

\section{THE RIEMANN ZETA FUNCTION AND THE RIEMANN HYPOTHESIS}

Prime numbers, the indivisible atoms of arithmetic, seem to be strewn haphazardly along the number line, starting with $2,3,5,7,11,13,17$ and continuing without pattern ad infinitum. But in 1859, the great German mathematician Bernhard Riemann hypothesized that the spacing of the primes logically follows from other numbers, now known as the "nontrivial zeros" of the Riemann zeta function.

The Riemann zeta function takes inputs that can be complex numbers - meaning they have both "real" and "imaginary" components - and yields other number$\mathrm{s}$ as outputs. For certain complex-valued inputs, the function returns an output of zero; these inputs are the "nontrivial zeros" of the zeta function. Riemann discovered a formula for calculating the number of primes up to any given cutoff by summing over a sequence of these zeros. The formula also gave a way of measuring the fluctuations of the primes around their typical spacing - how much larger or smaller a given prime was when compared with what might be expected.

The Riemann zeta function $\zeta(s)$ is a function of a complex variable $s=x+i y$, where $x$ and $y$ are real numbers and $i$ is the usual imaginary unit. The following infinite series converges for all complex numbers $s$ with real part

*Electronic address: sunb@cput.ac.za greater than 1 , and defines $\zeta(s)$ in this case:

$$
\zeta(s)=\sum_{n=1}^{\infty} \frac{1}{n^{s}}=\frac{1}{1^{s}}+\frac{1}{2^{s}}+\frac{1}{3^{s}}+\cdots .
$$

Via analytic continuation, one can show that: $\zeta(-1)=$ $-\frac{1}{12}, \zeta(0)=-\frac{1}{2}, \zeta(1)=\infty$, and $\zeta(2)=\frac{\pi^{2}}{6}$.

Bernhard Riemann [1-3], who was the first to apply the tools of complex analysis to this function in Eq.(1), proved that the function defined by the infinite summation (Riemann, 1859) can be analytically continued over the complex $s$ plane, except for $s=1$. This analytic continuation of the function is called the Riemann-zeta function. Riemann also derived a functional equation, containing the $\zeta(s)$ function, which is valid for all complex s and exhibits mirror symmetry around the $x=1 / 2$ vertical line, called the critical line. Figure 1 depicts the pole and zero structure of $\zeta(s)$ on the complex $s$ plane including the possible zeros off the critical line.

So far the statements about the zeros of $\zeta(s)$ and their locations on the complex plain were simple. However the distribution of the non-trivial zeros holds one of the most intriguing and enigmatic mathematical mysteries of the last century and a half. It is embarrassingly easy to pose Riemann' s conjecture:

The Riemann Conjecture All non-trivial zeros of $\zeta(s)$ have the form $s=1 / 2+i y$, where $y$ is a real number.

In other words all non-trivial zeros lie on the critical line. In 1900 Hilbert nominated the Riemann Hypothesis as the eighth problem on his famous list of compelling problems in mathematics (Hilbert, 1902). Since then not just professional mathematicians but mathematical soldiers of fortune tried, and still try, to verify its validity. The stakes are high. Whoever proves or disproves this 


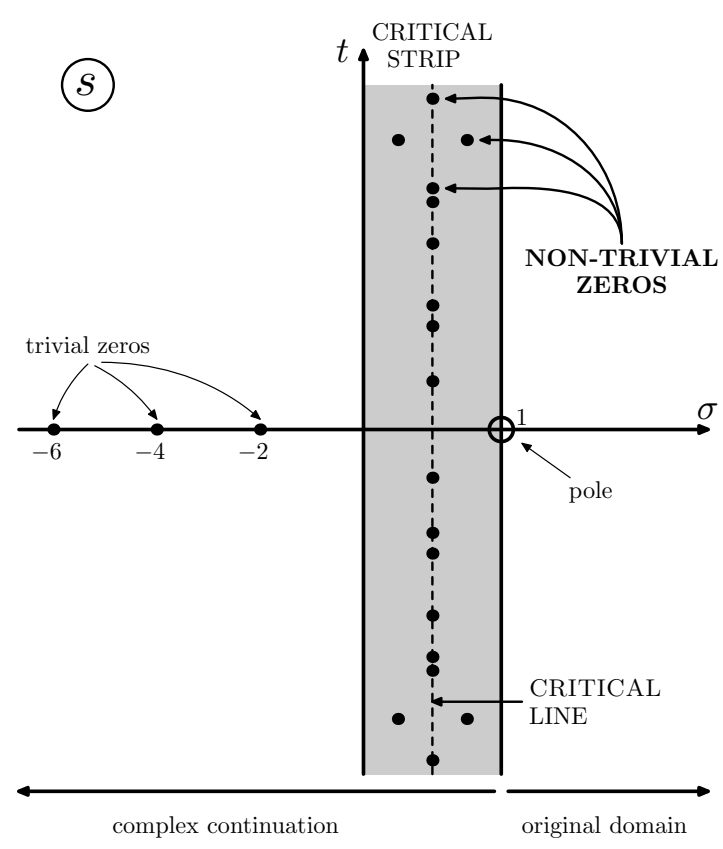

FIG. 1: The Riemann Zeta On Complex Plane

hypothesis engraves his name in the tablets of the history of mathematics, and may also receive one million dollars from the Clay Mathematics Institute [4].

\section{ASSEMBLING SPRINGS}

In mechanics, two or more springs are said to be in series when they are connected end-to-end, so as to act as a single spring. More generally, two or more springs are in series when any external stress applied to the ensemble gets applied to each spring without change of magnitude, and the amount strain (deformation) of the ensemble is the sum of the strains of the individual springs [6].
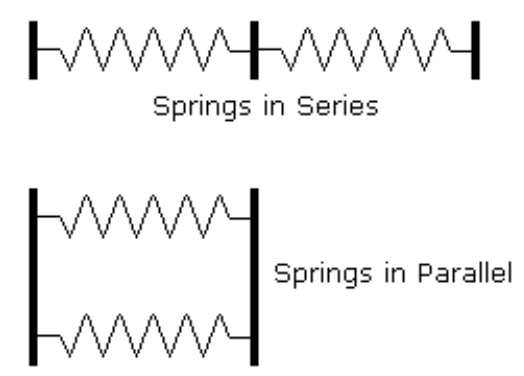

FIG. 2: Spring in both series and parallel

Equivalent Spring Constant (Series): When putting two springs in their equilibrium positions in series attached at the end to a block and then displacing it from that equilibrium, each of the springs will experience corresponding displacements $d_{1}$ and $d_{2}$ for a total displacement of $d_{1}+d_{2}$. For the spring that is equivalent to a system of two springs, in series, whose spring constants are $k_{1} k_{2}$. The total equivalent stiffness is given by $\frac{1}{K}=\frac{1}{k_{1}}+\frac{1}{k_{2}}$. If the spring system has infinite springs, the total equivalent stiffness is given by

$$
\frac{1}{K}=\frac{1}{k_{1}}+\frac{1}{k_{2}}+\cdots+\frac{1}{k_{n}}+\cdots=\sum_{n=1}^{\infty} \frac{1}{k_{n}} .
$$

The compressed force has a relation $k_{1} d_{1}=k_{2} d_{2}=$ $\cdots=k_{n} d_{n}=\cdots=$ const. $=\alpha$.

The compressed distances for the in series case is $d=$ $d_{1}+d_{2}+\cdots=\alpha \sum_{n=1}^{\infty} \frac{1}{k_{n}}$.

Energy stored for the n-th spring is $E_{n}=\frac{1}{2} k_{n} d_{n}^{2}$. For the series case, the ratio of energy stored in springs $\frac{E_{1}}{E_{2}}=$ $\frac{1 / 2 k_{1} d_{1}^{2}}{1 / 2 k_{2} d_{2}^{2}}=\frac{k_{2}}{k_{1}}=\frac{d_{1}}{d_{2}}$.

Total energy of the spring is given by

$$
E=\sum_{n=1}^{\infty} E_{n}=\frac{1}{2} \sum_{n=1}^{\infty} k_{n} d_{n}^{2}=\frac{1}{2} \alpha^{2} \sum_{n=1}^{\infty} \frac{1}{k_{n}}=\frac{1}{2} \frac{\alpha^{2}}{K} .
$$

\section{THE RIEMANN SPRING IN SERIES}

Comparing the two expression Eq.1 and Eq.2, it is easy to notice that they are similar. If the spring system has an infinite springs in series, whose $n$-th spring has elastic complex modulus $k_{n}=n^{s}$, therefore total equivalent modulus $K(s)$ is given by

$$
\frac{1}{K(s)}=\sum_{n=1}^{\infty} \frac{1}{k_{n}}=\sum_{n=1}^{\infty} \frac{1}{n^{s}}=\zeta(s) .
$$

where the Riemann Zeta function is $\zeta(s)=\sum_{n=1}^{\infty} \frac{1}{n^{s}}$. It is clear that the Riemann spring stiffness $K(s)$ is a complex which is different from the physical spring, whose stiffness is real number.

From the previous section, we have following relations for the Riemann spring in series:

The compressed force has a relation $1^{s} d_{1}=2^{s} d_{2}=$ $\cdots=n^{s} d_{n}=\cdots=\alpha$.

The Riemann spring elastic energy for the n-th spring is $E_{n}=\frac{1}{2} \frac{1}{n^{s}} d_{n}^{2}$.

Total energy of the Riemann spring is given by

$$
E=\sum_{n=1}^{\infty} E_{n}=\frac{1}{2} \sum_{n=1}^{\infty} n^{s} d_{n}^{2}=\frac{\alpha^{2}}{2} \sum_{n=1}^{\infty} \frac{1}{n^{s}}=\frac{1}{2} \alpha^{2} \zeta(s)
$$

which reveals that the total energy of the Riemann spring is proportional to the Riemann Zeta function. 
For complex number $s=x+i y$, thus $n^{s}=$ $n^{(x+i y)}=n^{x} n^{i y}=n^{x} \exp [(i \ln n) y]=n^{x}[\cos (y \ln n)+$ $i \sin (y \ln n)$ ], we have $\Re\left(n^{s}\right)=n^{x} \cos (y \ln n)$ and $\Im\left(n^{s}\right)=$ $n^{x} \sin (y \ln n)$.

The stiffness for the $\mathrm{n}$-th spring is $k_{n}=n^{s}=$ $n^{(x+i y)}$, therefore, $\Re\left(k_{n}\right)=n^{x} \cos (y \ln n)$ and $\Im\left(k_{n}\right)=$ $n^{x} \sin (y \ln n)$.

The reciprocal of $k_{n}$ is

$$
\begin{aligned}
\frac{1}{k_{n}} & =\frac{1}{n^{s}}=\frac{1}{n^{(x+i y)}}=n^{-(x+i y)}=n^{-x} n^{-i y} \\
& =n^{-x}[\cos (y \ln n)-i \sin (y \ln n)] .
\end{aligned}
$$

Thus the total stiffness of the Riemman spring in series is

$$
\frac{1}{K}=\sum_{n=1}^{\infty} \frac{1}{k_{n}}=\Re\left(\frac{1}{K}\right)+i \Im\left(\frac{1}{K}\right) .
$$

where $\Re\left(\frac{1}{K}\right)=\sum_{n=1}^{\infty} n^{-x} \cos (y \ln n)$ and $\Im\left(\frac{1}{K}\right)=$ $-\sum_{n=1}^{\infty} n^{-x} \sin (y \ln n)$.

The total energy can be rewritten as

$$
\begin{aligned}
& E=\frac{1}{2} \alpha^{2} \zeta(s) \\
& =\frac{\alpha^{2}}{2} \sum_{n=1}^{\infty} n^{-x} \cos (y \ln n)-i \frac{\alpha^{2}}{2} \sum_{n=1}^{\infty} n^{-x} \sin (y \ln n)
\end{aligned}
$$

\section{THE RIEMANN HYPOTHESIS WITHIN THE FRAMEWORK OF CLASSIC SPRING MECHANICS}

Using the Riemann spring model, the Riemann conjecture, namely, all non-trivial zeros of $\zeta(1 / 2+i y)$ can be expressed in terms of total stiffness as follows:

$$
\frac{1}{K(1 / 2+i y)}=\left.\zeta\left(\frac{1}{2}+i y\right)\right|_{y=r_{k}}=0
$$

where $r_{k}$ is the $\mathrm{k}$-th point that the Riemann Zeta function vanishes. Physically speaking, this condition means that the total Riemann stiffness $K\left(\frac{1}{2}+i y\right)$ is infinite at points $r_{k}$.

Considering the Eq.(9) as a constraint to the Riemann spring, the modified Lagrange $L$ can be constructed as follows

$$
\begin{aligned}
L & =E+\lambda \zeta\left(\frac{1}{2}+i y\right)=\frac{1}{2} \alpha^{2} \zeta\left(\frac{1}{2}+i y\right) \\
& +\left.\lambda \zeta\left(\frac{1}{2}+i y\right)\right|_{y=r_{k}} .
\end{aligned}
$$

where $\lambda$ is the Lagrange multiplier, which can determined by the variational $\delta L=0$, namely

$$
\begin{aligned}
\delta L & =\frac{1}{2} \alpha^{2} \frac{d \zeta}{d s} i \delta y+\left.\lambda \frac{d \zeta}{d s}\right|_{y=r_{k}} i \delta y \\
& +\left.\delta \lambda \zeta\left(\frac{1}{2}+i y\right)\right|_{y=r_{k}}=0 .
\end{aligned}
$$

which will give following relations $\left.\zeta\left(\frac{1}{2}+i y\right)\right|_{y=r_{k}}=0$ and $\lambda=-\frac{1}{2} \alpha^{2} \frac{d \zeta}{d s} /\left.\frac{d \zeta}{d s}\right|_{y=r_{k}}$. Substitute this to Eq.12 will lead a generalized variational as fllows

$$
\begin{aligned}
L & =\frac{1}{2} \alpha^{2} \zeta\left(\frac{1}{2}+i y\right) \\
& +\left.\frac{1}{2} \frac{d \zeta}{d s} \frac{\alpha^{2}}{\left.\frac{d \zeta}{d s}\right|_{y=r_{k}}} \zeta\left(\frac{1}{2}+i y\right)\right|_{y=r_{k}} .
\end{aligned}
$$

\section{RIEMANN SPRING-MASS SYSTEM FREQUENCY}

If the Riemann spring in series has a central mass $M$, the spring-mass system will be governed by

$$
M \frac{d^{2} x}{d t^{2}}+K x=f(t)
$$

We can get its frequency $\omega=\sqrt{\frac{K}{M}}=\frac{1}{\sqrt{M \zeta}}$, which is a complex number.

\section{THE RIEMANN SPRING IN PARALLEL}

For the Riemann spring in parallel, the equivalent stiffness is given by $K(s)=\sum_{n=1}^{\infty} k_{n}=\sum_{n=1}^{\infty} n^{s}$, and displacements $d_{1}=d_{2}=\cdots=d_{n}=\cdots$, forces $F=$ $F_{1}+F_{2}+\ldots$, energy relations $\frac{E_{1}}{E_{2}}=\frac{k_{1}}{k_{2}}=\cdots=\frac{k_{n}}{k_{n+1}} \cdots$.

\section{CONCLUSIONS}

The Riemann spring proposed here is just analogue to the classis spring in series. All relevant analogue quantities are complex instead of real one. This pure mathematical analogy does not take into account all quantities physical dimensions. 
Riemann Hypothesis, Rev. Mod. Phys. 83:307-330,2011 DOI:10.1103/RevModPhys.83.307

[3] Hardy, G. H., and Wright, E. M., 1960, An Introduction to the Theory of Numbers (Clarendon Press) 4th edition.

[4] https://en.wikipedia.org/wiki/Riemann_hypothesis
[5] Chen, N.X. Modified Möbius inverse formula and its applications in physics [J]. Phys.Rev.Lett. 64, 1193, 1990.

[6] https://en.wikipedia.org/wiki/Series_and_parallel_springs 